\title{
Parameters of an Unique Condenson State in the Structure of the $\mathrm{In}_{4} \mathrm{Se}_{3}$ Crystal
}

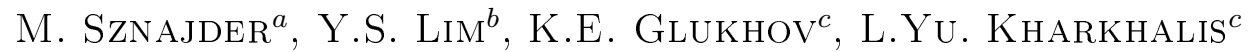 \\ AND D.M. BERCHA ${ }^{a, c}$ \\ ${ }^{a}$ Institute of Physics, University of Rzeszów, T. Rejtana 16a, 35-310 Rzeszów, Poland \\ ${ }^{b}$ Green Ceramics Division, Korea Institute of Ceramic Engineering and Technology \\ 233-5 Gasan-dong, Geumcheon-gu, Seoul 153-801, Korea \\ ${ }^{c}$ Institute of Physics and Chemistry of Solid State, Uzhgorod National University, Ukraine \\ Based upon the $a b$ initio band structure calculations results the dispersion law parameters of charge carriers \\ of the orthorhombic $\mathrm{In}_{4} \mathrm{Se}_{3}$ semiconductor as well as of its Sn- and Te-doped compounds were calculated. This \\ allowed to estimate parameters of the electron condenson states in those compounds.
}

PACS: 71.15.Mb, 71.20.-b, 71.20.Nr, 71.38.-k

\section{Introduction}

After the discovery of excellent thermoelectric properties of the single crystalline rhombic semiconductor $\mathrm{In}_{4} \mathrm{Se}_{3}$ quantified by the dimensionless figure of merit, $Z T$, which appeared to be larger than 1.4 [1], an intensive research of the indium selenides group has re-started. To the thermoelectric performance of a material, governed by the $Z T$ value, contribute both electrical conductivity $\sigma$, the Seebeck coefficient $\alpha$ and thermal conductivity $\kappa$. The chalcogenide indium semiconductors with layered structure are potential thermoelectric candidates due to their low thermal conductivity. Since there is a possibility to interpret the thermoelectric properties of $\mathrm{In}_{4} \mathrm{Se}_{3}$ at high temperatures [2] by means of the condenson state theory [3], it is necessary to re-examine first the parameters of its dispersion law for charge carriers.

\section{Parameters of the condenson state in $\mathrm{In}_{4} \mathrm{Se}_{3}$, Sn- and Te-doped $\operatorname{In}_{4} \mathrm{Se}_{3}$ semiconductors}

The concept of condenson was introduced by Deigen and Pekar [3] who analyzed the interaction of a conduction electron with acoustic phonons in a homopolar dielectric within the effective mass and potential deformation approximations, for a quadratic dispersion law governing the electron motion. A functional of a total energy of the system is

$$
\begin{aligned}
& E\left[\psi, \varepsilon_{i j}\right]=\frac{\hbar^{2}}{2 m^{*}} \int(\nabla \psi)^{2} \mathrm{~d} \boldsymbol{r}+\int \sum_{i j} b_{i j} \varepsilon_{i j}|\psi|^{2} \mathrm{~d} \boldsymbol{r} \\
& \quad+U\left(\left\{\varepsilon_{i j}\right\}\right),
\end{aligned}
$$

where $b_{i j}$ are the deformation potential tensor components and $\varepsilon_{i j}$ are the deformation tensor components.
Second term in (1) describes interaction of an electron with lattice, while the third one is the energy of a local elastic deformation of crystal. By varying (1) with respect to deformation tensor components it is possible to connect those components with the elastic constants and wave function $\psi$ of the system. As a result, functional (1) takes the form

$$
E[\psi]=\frac{\hbar^{2}}{2 m^{*}} \int(\nabla \psi)^{2} \mathrm{~d} \boldsymbol{r}-\frac{b^{2}}{\lambda} \int|\psi|^{4} \mathrm{~d} \boldsymbol{r},
$$

where $b$ and $\lambda$ represent combinations of deformation potential tensor components and elastic moduli, respectively. Since the bounded electron states are expected to arise in a crystal, the probe function $\psi$ can be chosen as [4]:

$$
\psi(\boldsymbol{r})=\mu^{d / 2} \psi_{0}(\mu \boldsymbol{r}),
$$

where $\mu$ is a variational parameter that connects the radius $r_{\mathrm{e}}$ of the condenson formation with the lattice constant $a\left(\mu \sim a r_{\mathrm{e}}^{-1}\right)$, and which is at the same time the parameter of a scale transformation. The scale transformation gives a possibility to analyze our issue for three cases of space dimension $(d=1,2,3[4])$. In the case of a 3 D-crystal $(d=3)$ and anisotropic scale transformation, the $\psi$ function takes the form $[5,6]$ :

$$
\begin{aligned}
& \psi(r)=\left(\frac{\left(\mu_{1} \mu_{2} \mu_{3}\right)^{1 / 3} \sqrt{2}}{\left(a_{1} a_{2} a_{3}\right)^{1 / 3}}\right)^{3 / 2} \\
& \quad \times \exp \left(-\pi\left(\left(\frac{\mu_{1} x}{a_{1}}\right)^{2}+\left(\frac{\mu_{2} y}{a_{2}}\right)^{2}+\left(\frac{\mu_{3} z}{a_{3}}\right)^{2}\right)\right) .
\end{aligned}
$$

Substitution of (3) into (2) leads to a functional in the form [4]: 


$$
E[\mu]=a_{1} \mu^{2}-a_{2} \mu^{d},
$$
where $a_{1}$ and $a_{2}$ are quantities in units of energy [3]. It can be shown that in a 3D case functional (5) has no minimum. Hence, the stable condenson states of electron do not exist in continuum approximation, but only such states with large radius are interesting from the viewpoint of prognosis of material thermoelectric properties. On the other hand, in a 1D case in continuum approximation the condenson states (or self-localized states [7]) are possible. It was also shown that this approximation allows the existence of condenson states in deformed 2D-structures [8]. Hence, it is obvious that the possibility for the localized condenson state to arise, depending on the dimension of space, is closely related to a different density of states function for the parabolic dispersion law in the case of $3 \mathrm{D}, 2 \mathrm{D}$, and $1 \mathrm{D}$-crystals.

\section{Results}

Our first semi-empirical pseudopotential band structure calculations of the bulk $\mathrm{In}_{4} \mathrm{Se}_{3}$ crystal [5] showed that both its valence and conduction band is described by a dispersion law that differs essentially from the parabolic one

$$
E(k)=-\alpha_{1} k_{x}^{2}-\alpha_{2} k_{y}^{2}-\alpha_{3} k_{z}^{2}+\beta_{1} k_{x}^{4}+\beta_{2} k_{y}^{4}+\beta_{3} k_{z}^{4}
$$

with coefficients showing the opposite sign and inequality $\beta_{i} \gg \alpha_{i}$. Additionally, a peak-like density of states function was obtained for the dispersion law $(6)[5,6]$ which was analogous to that of $1 \mathrm{D}$-crystal with a parabolic dispersion law. The above type of dispersion law with negative curvature was confirmed also by $a b$ initio DFT based band structure calculations [9]. Such peculiarity of the $\mathrm{In}_{4} \mathrm{Se}_{3}$ spectrum is correlated well with the obtained by us peak of imaginary part of the dielectric permittivity tensor at the edge of its energy dependence, for the polarization of light in the direction (100) (see Fig. 1).

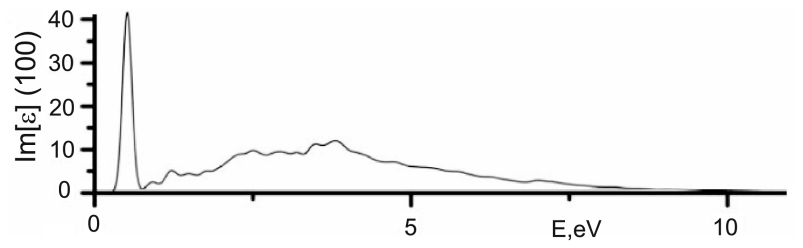

Fig. 1. The energy dependence of $\varepsilon_{2}$ for the polarization of light $\boldsymbol{E} \| \boldsymbol{a}$ in the $\mathrm{In}_{4} \mathrm{Se}_{3}$ crystal.

The coefficients of the dispersion law (6) are crucial to find the parameters of the condenson state. Hence, in order to correlate theoretical investigations with experimental data concerning thermoelectric properties of $\mathrm{In}_{4} \mathrm{Se}_{3}(\alpha(T), \sigma(T), \kappa(T)$ dependences), it is necessary to state first the coefficients of the dispersion law (6) precisely. The repeated $\mathrm{In}_{4} \mathrm{Se}_{3}$ electronic spectrum calculations with the use of other approaches and package programs (ab initio full-potential nonorthogonal local- -orbital minimum basis scheme and pseudopotential linear combination of atomic orbitals (LCAO) one) led to slightly different values of $\alpha_{i}$ and $\beta_{i}$. Spin-orbit interaction taken into account did not result in changes of the electronic spectrum, since the $\mathrm{In}_{4} \mathrm{Se}_{3}$ crystal is a centrosymmetric one and one-dimensional representations describing energy states (near $\Gamma$ point) were doubled only. In order to estimate changes of the dispersion law parameters due to the introduction of Sn and Te dopants to the host material, we calculated dispersion relations $E(k)$ for the $1 \times 1 \times 4 \mathrm{In}_{4} \mathrm{Se}_{3}$ supercell, both for pure crystal and with the presence of a pair of dopant atoms (see Fig. 2).
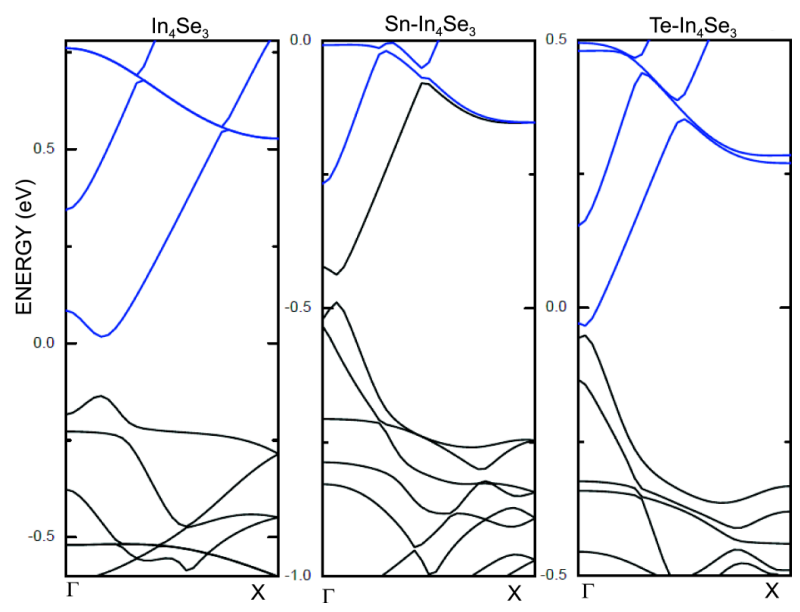

Fig. 2. Dispersion relations in the $\Gamma-X$ direction of the undoped (left), Sn-doped (middle), and Te-doped $\mathrm{In}_{4} \mathrm{Se}_{3}$ crystal.

The application of supercell allowed to model $\approx 3 \%$ and $\approx 4 \%$ dopant concentration of Sn and Te, respectively. Introduction of Sn dopant pair is motivated by a necessity to obtain the semiconducting type of band occupation. The band structure calculations were done in the framework of density functional theory (DFT) with the use of local density approximation (LDA) to describe exchange-correlation interaction, by means of the package program SIESTA [10]. Since the periodic boundary conditions were applied for the cell's modeling they led to a uniform placement of dopants in every supercell, which is to some extent a kind of idealization. It should be also noted that due to a large electronegativity of Te atoms, the wave functions of neighboring dopants overlap to a smaller extent than those of Sn atoms, and hence, the results obtained for the Te dopants are more adequate. As a consequence, the introduction of two Sn dopant atoms having four valence electrons instead of two trivalent In atoms leads to the occupation of another extra band and to a change of conductivity type from the semiconducting to metallic one. Certainly, at the chaotic placement of dopants a local level should appear which would smear to a dopant band in the energy gap with the increase in dopant concentration. Nevertheless, it is possible to 
investigate changes of the dispersion law (6) parameters by comparing the corresponding bands of the doped and host materials. In the case of isovalent Te dopant, the number of dispersion bands remains unchanged and the obtained results can be compared directly to the results of a host $\mathrm{In}_{4} \mathrm{Se}_{3}$ material.

Coefficients of the dispersion law (6)

TABLE and parameters of the condenson state.

\begin{tabular}{|c|c|c|c|c|}
\hline & \multirow[b]{2}{*}{$\operatorname{In}_{4} \mathrm{Se}_{3}[5,6]$} & \multicolumn{3}{|c|}{ Supercell $1 \times 1 \times 4$} \\
\hline & & $\mathrm{In}_{4} \mathrm{Se}_{3}$ & $\begin{array}{c}\mathrm{In}_{4} \mathrm{Se}_{3-x} \mathrm{Te}_{x} \\
(x=0.125)\end{array}$ & $\begin{array}{c}\operatorname{In}_{4-x} \mathrm{Se}_{x} \mathrm{Se}_{3} \\
(x=0.125)\end{array}$ \\
\hline$\alpha_{1}[\mathrm{eV}]$ & 5.7 & 4.0 & 7.16 & 6.24 \\
\hline$\alpha_{2}$ & 13.0 & -0.46 & 0.72 & -0.14 \\
\hline$\alpha_{3}$ & 3.1 & -0.52 & -0.08 & -0.35 \\
\hline$\beta_{1}$ & 479.8 & 59.9 & 691.33 & 610.4 \\
\hline$\beta_{2}$ & 888.0 & 3.54 & 13.81 & 9.91 \\
\hline$\beta_{3}$ & 2957 & 0.50 & 1.51 & 1.42 \\
\hline$E_{1}[\mathrm{eV}]$ & -0.01 & -0.012 & -0.0071 & -0.0044 \\
\hline$r_{\mathrm{e}}[\AA]$ & 100 & 52 & 107.8 & 117.50 \\
\hline
\end{tabular}

The calculated values of $\alpha$ and $\beta$ coefficients for all three discussed cases are summarized in Table. Additionally, for a comparison, we include parameters of the dispersion law (6) obtained by means of semi-empirical pseudopotential band structure calculations [5] together with the condenson state parameters, i.e. its binding energy $E_{1}$ and radius $r_{\mathrm{e}}$. From Table it follows that the tendency to preserve non-parabolic behavior of dispersion relations is kept for the $\mathrm{Sn}$ - and Te-doped $\mathrm{In}_{4} \mathrm{Se}_{3}$. The non-parabolic dispersion relation is a necessary condition for the condenson states to exist in those materials. At the same time, introduction of Te dopant leads to a change of sign of the quadratic term coefficient in the $\Gamma-Y$ direction, which is observed neither in the case of undoped nor $\mathrm{Sn}$-doped $\mathrm{In}_{4} \mathrm{Se}_{3}$. Investigations on the condenson state dynamics show that introduction of a dopant into cationic or anionic sublattices increases the effective radius of these collective excitations, which can lead to a "multicondenson" state $[5,6]$. However, is should be noted that all the obtained results were obtained at $T=0 \mathrm{~K}$ and they are estimations of the situation that take place at elevated temperatures.

\section{References}

[1] J.-S. Rhyee, K.H. Lee, S.M. Lee, E. Cho, S.I. Kim, E. Lee, Y.S. Kwon, J.H. Shim, G. Kotliar, Nature 459, 965 (2009).

[2] Y.S. Lim, J.Y. Cho, J.-K. Lee, S.-M. Choi, Electron. Mater. Lett. 6, 117 (2010).

[3] M.F. Deigen, S.I. Pekar, Zh. Eksp. Teor. Fiz. 21, 803 (1951).

[4] V.A. Kochelap, V.N. Sokolov, B.Yu. Vengalis, Phase Transitions in Semiconductors with Deformation Electron-Phonon Interaction, Naukova Dumka, Kiev 1984.

[5] D.M. Bercha, L.Yu. Kharkhalis, A.I. Bercha, M. Sznajder, Phys. Status Solidi B 203, 427 (1997).

[6] D.M. Bercha, L.Yu. Kharkhalis, A.I. Bercha, M. Sznajder, Phys. Techn. Semicond. 37, 1299 (1997).

[7] Yu. Toyozawa, Prog. Theor. Phys. 26, 29 (1961).

[8] O.B. Kibis, Phys. Techn. Semicond. 29, 125 (1995).

[9] M. Sznajder, K.Z. Rushchanskii, L.Yu. Kharkhalis, D.M. Bercha, Phys. Status Solidi B 243, 592 (2006).

[10] http://www.icmab.es/siesta/. 